\title{
Re: Treatment of non-union of humerus diaphyseal fractures
}

\author{
Yueju Liu $\cdot$ Han Li
}

Received: 3 June 2014/Published online: 10 August 2014

(C) Springer-Verlag Berlin Heidelberg 2014

To the Editor,

Singh AK and colleagues [1] have done an interesting study in the treatment of non-union of humerus diaphyseal fractures through comparing of interlocking nail and locking compression plate, which found both the implants can be used in non-union of humeral shaft fractures with good functional outcomes and acceptable rate of complications. However, we have some concerns regarding the paper and wish to share them.

In the Figure 2 of this article, figure $\mathrm{a}$ and $\mathrm{b}$ showed non-union of humeral diaphyseal fracture in 30-year-old female with implant in situ, then removal of implant, freshening of fracture edges, internal fixation with antegrade interlocking nail and autogenous iliac crest bone grafting was done, which showed us a X-ray of non-union of fracture 15-months later. However, it was typical hypertrophic non-union, which referred to as a hypervascular and viable non-union [2]. Such type of non-union was caused mainly by lack of adequate immobilization, which was a main fallback of intramedullary nailing of humeral shaft fracture. Then, in the following Revision surgery of removal of nail, freshening of fracture edges, internal fixation with LCP and autogenous iliac crest bone grafting,

\section{Y. Liu $(\bowtie) \cdot$ H. Li}

Department of Orthopedic Center, Third Hospital of Hebei Medical University, No. 139 Zi Qiang Road,

Shijiazhuang 050051, Hebei, People's Republic of China e-mail: liuyueju1983@gmail.com

\section{H. Li}

e-mail: lihandoctor@126.com

\section{Y. Liu $\cdot$ H. Li}

Key Orthopaedic Biomechanics Laboratory of Hebei Province, Shijiazhuang, People's Republic of China the fracture got good union in the Figure c. This typical figure suggest us that plate osteosynthesis remains the criterion standard of fixation of humeral shaft fractures against intramedullary nailing. In our opinion, intramedullary is restricted to pathologic fracture [3] and fractures with poor soft tissue over the fracture site such as burns.

Second, another main drawback in this study was lack of classification of non-union. As we know, there were three types of non-union, such as atrophic, oligotrophic and hypertrophic. So, it was a prerequisite that there was no statistical difference between the groups in the type of nonunion before comparing of interlocking nail and locking compression plate. However, it was just missing in this article. So, we want to know the missed content and advise that the locking compression plate should be the first choice for hypertrophic non-union.

Conflict of interest The authors confirm that there are no known conflicts of interest associated with this publication and that there has been no significant financial supports for this work that could have influenced its outcome.

\section{References}

1. Singh AK, Arun GR, Narsaria N, Srivastava A (2014) Treatment of non-union of humerus diaphyseal fractures: a prospective study comparing interlocking nail and locking compression plate. Arch Orthop Trauma Surg. (Epub ahead of print)

2. Lin J, Shen PW, Hou SM (2003) Complications of locked nailing in humeral shaft fractures. J Trauma 54:943-949

3. Redmond BJ, Biermann JS, Blasier RB (1996) Interlocking intramedullary nailing of pathological fractures of the shaft of the humerus. J Bone Jt Surg Am 78:891-896 